\title{
Haciendo gelatinas: dos situaciones didácticas sobre la creación y uso de registros con niños preescolares
}

\author{
Making Jellies: Two didactic situations about the creation \\ and use of registers with preschool children
}

\author{
María Laguna ${ }^{1}$ \\ David Block Sevilla²
}

\begin{abstract}
Resumen: En este artículo analizamos dos situaciones didácticas relativas al tema de Tratamiento de la Información, así como algunos resultados de su implementación en dos grupos de preescolar. Una de las situaciones propicia la representación de objetos y acciones necesarios para elaborar un producto (gelatinas), la otra enfrenta a los alumnos a la necesidad de obtener información de otro grupo de alumnos, también, con la finalidad de elaborar gelatina, pero esta vez para otros. Ambas situaciones dieron lugar a una rica gama de producciones de los alumnos y propiciaron cierta evolución de éstas. Tanto el diseño como la implementación estuvieron orientados por un enfoque constructivista de la enseñanza. Además, nos interesó que las situaciones fueran comprensibles y manejables para las educadoras. Esta última característica sitúa al estudio como una experiencia de Ingeniería Didáctica de Desarrollo. ${ }^{3}$
\end{abstract}

Fecha de recepción: 01 de agosto de 2019. Fecha de aceptación: 15 de octubre de 2020.

${ }^{1}$ Departamento de Investigaciones Educativas del Centro de Investigación y Estudios Avanzados (DIE-CINVESTAV-IPN), México, CDMX, columbus_mgrl@hotmail.com, orcid.org/0000-0002-6104-6593

${ }^{2}$ Departamento de Investigaciones Educativas del Centro de Investigación y Estudios Avanzados (DIE-CINVESTAV-IPN), México, CDMX, dblock@cinvestav.mx, orcid.org/0000-0002-3914-5544

${ }^{3}$ Agradecemos a las educadoras Liliana Escobar, Beatriz Vega y Eréndira Alarcón por su participación en este estudio, y a nuestras colegas Laura Reséndiz y Margarita Ramírez por sus valiosas observaciones. 
Palabras clave: educación preescolar, enseñanza de las matemáticas, tratamiento de la información, estadística.

\begin{abstract}
In this article, we analyze two didactic situations related to the thematic of Data Analysis, as well as some results of its implementation in two preschool groups. One of the situations conduces to the representation of objects and actions necessary to develop a product (jellies), the other confronts students with the need to obtain information from another group of students, with the same purpose. Both situations gave rise to a rich range of student productions and led to some evolution of these. The design and implementation were guided by a constructivist approach to teaching. Furthermore, it interested that the situations were understandable and manageable for educators. This last characteristic situates the study as an experience of Didactic Development Engineering.
\end{abstract}

Keywords: preschool education, mathematics education, data analysis, statistics.

\title{
1. INTRODUCCIÓN
}

Cada vez más, los niños están inmersos en situaciones cotidianas pobladas de datos, lo que les brinda la posibilidad de usar conocimientos matemáticos vinculados a su entorno e integrarlos con los de otras disciplinas, como lengua o ciencias. Al mismo tiempo que se ofrecen estas oportunidades, se crean exigencias: interpretar y representar información constituyen necesidades para la vida en sociedad. En este sentido, es previsible que los contenidos de Tratamiento de la Información, irán encontrando más espacio en la escuela, desde edades cada vez más tempranas.

El propósito de este artículo es contribuir al desarrollo de situaciones didácticas de tratamiento de la información (o estadística), dirigidas al nivel de preescolar y orientadas por un enfoque constructivista, tomando en consideración su factibilidad para ser implementadas por los docentes en condiciones normales de trabajo. 


\section{1 EL TRATAMIENTO DE LA INFORMACIÓN EN EL CURRÍCULO}

En México, los contenidos de Probabilidad y Estadística se introdujeron en primaria y secundaria con la reforma de los años 70 (SEP, 1972). En preescolar, con la reforma de 2004, aparece por primera vez de manera explícita en el campo de pensamiento matemático, donde se enuncia la siguiente competencia: "Reúne información sobre criterios acordados, representa gráficamente dicha información y la interpreta" (SEP, 2004: 77). En el modelo educativo vigente, el tratamiento de la información se consolida desde preescolar hasta secundaria, bajo el nombre de Análisis de Datos (SEP, 2018: 314). Durante los tres años de educación preescolar, se propone que los niños recopilen y registren datos en tablas o gráficas a partir de preguntas que planteen una situación a resolver (SEP, 2018: 306).

A partir de una revisión de programas y propuestas didácticas de diferentes países (Laguna, 2016), hemos categorizado en seis grupos los tipos de tarea ${ }^{4}$ más recurrentes en el eje de Tratamiento de la Información: 1) clasificar objetos a partir de diferentes criterios (rojos y grandes, por ejemplo), o bien, definir los criterios a partir de una clasificación ya hecha, 2) recopilar datos cualitativos y/o cuantitativos para organizarlos en registros, 3) representar información en gráficas, 4) interpretar y analizar datos de gráficas, cuadros, diagramas o imágenes, 5) tomar decisiones razonadas con base en información analizada previamente, y 6) diferenciar sucesos seguros, de posibles e imposibles, y comparar la probabilidad de sucesos aleatorios.

Respecto a las prácticas reales de enseñanza del tratamiento de la información en preescolar, identificamos solamente un estudio (Fuenlabrada, 2010) en el que se documenta y analiza la tendencia a dejar este eje de lado en las aulas. Se muestra que, en los casos en que se atiende, se le reduce a experiencias de conteo. Con la experiencia que comunicamos a continuación, buscamos contribuir a disminuir este vacío. Algunos aportes específicos, esta vez desde la investigación, serán mencionados al analizar las situaciones.

${ }^{4}$ Tomamos el término de tarea en el sentido que le da Chevallard (1991); una tarea es la acción sobre un objeto particular, mientras que, un tipo de tareas es la acción que puede recaer sobre un diverso tipo de objetos. 


\subsection{EL ENFOQUE CONSTRUCTIVISTA Y LA ATENCIÓN AL DOCENTE}

El enfoque constructivista promovido en el currículo mexicano, y en el de muchos otros países, desde hace al menos 30 años, se caracteriza por la tesis según la cual los alumnos pueden aprender matemáticas de manera significativa mediante la interacción con situaciones que les plantean un problema, un reto. Para dar lugar a dichos procesos de aprendizaje, los problemas no pueden ser cualesquiera, deben reunir determinadas características, al igual que la gestión docente.

En el último medio siglo se ha desarrollado la Teoría de las Situaciones Didácticas (Brousseau, 1986) que explora alternativas de enseñanza de las matemáticas, y que especifica las características deseadas en los problemas para que sean susceptibles de propiciar construcción de conocimientos matemáticos. En esta teoría se ha acuñado el término de situación adidáctica (Brousseau, 2007) para referirse a una situación que, en principio, hace posible que el alumno elabore determinados conocimientos por sí mismo, eventualmente en colaboración con sus pares. Del docente, se espera cierta reticencia (Sensevy, 2011) a guiar, a proporcionar respuestas, durante los momentos de construcción. Las características básicas de tales situaciones son: 1) implicar al conocimiento que interesa, 2) ser accesible inicialmente sin disponer aún de dicho conocimiento, 3) permitir al aprendiz mismo comprobar si sus intentos de resolución realizados fueron exitosos o no, esto es, dar lugar a una forma de validación, lo anterior supone que se puede lograr un compromiso intelectual del alumno con el medio $^{5}$ (Sadovsky, 2005).

Sin embargo, no solamente ha sido complicado disponer de las situaciones didácticas adecuadas para funcionar como adidácticas y favorecer el aprendizaje de los diferentes conocimientos de matemática, también lo ha sido utilizar aquellas diseñadas en el marco de la investigación. Estudios sobre prácticas docentes realizados en los últimos 20 años (Robert, 2003; Block et al., 2008) señalan dificultades que han tenido los profesores al implementar dichas situaciones en las aulas. Entre estas dificultades, destaca la de permitir que los alumnos resuelvan los problemas con autonomía, dando validez a los conocimientos informales, parciales o erróneos que se generan en los procesos de

5 Medio, en el sentido de la TSD, refiere a la problemática a la que se enfrenta al alumno, incluyendo el problema y las condiciones que se dan para resolverlo. Es un sentido cercano al que tiene en la epistemología genética. 
aprendizaje. El que estas dificultades fueran detectadas tardíamente (en relación con el desarrollo de los conocimientos de la didáctica) se atribuye en parte a que, en una primera etapa, las investigaciones realizadas en el marco de la TSD se centraron en los aprendizajes de los alumnos en función de las situaciones, quedando en segundo plano la problemática docente (Artigue, 2011).

Los señalamientos anteriores han dado lugar a líneas de estudio que, sin dejar de lado los aportes de las investigaciones didácticas anteriores, recuperan la centralidad del docente en la gestión de las situaciones. En particular, se propone una metodología llamada Ingeniería Didáctica de Desarrollo (Perrin-Glorian, 2011) de la que retomamos varios elementos en nuestro estudio.

\subsection{METOdOLOGÍA Y PREGUNTAS DE INVESTIGACIÓN}

La investigación más amplia que enmarca la implementación de las dos situaciones didácticas que analizaremos, tuvo como propósito estudiar procesos de apropiación de situaciones didácticas por parte de docentes en servicio (Laguna, 2016). En el presente artículo, cambiamos el foco de la mirada para centrarlo en las interacciones de los alumnos con dichas situaciones -la receta y la encuesta-. Para ello, analizamos los resultados de la experimentación en aula, destacando el papel de las situaciones en las producciones de los niños y en sus posibles aprendizajes. Previamente, mediante un análisis $a$ priori (Artigue, 1995) y con el auxilio de categorías de las TSD (Brousseau, 1986), señalamos algunas características didácticas de ambas situaciones, empezando por la forma en que se implican los conocimientos que son objeto de estudio.

Las situaciones que analizamos forman parte de una secuencia que denominamos Haciendo gelatinas. Dicha secuencia emana, en su versión original, del relato de un docente de preescolar que hace encuestas con sus alumnos para hacer gelatinas (SEP, 2010). Nos pareció que la idea presentaba un interés particular para ser estudiada empíricamente, empezando por el hecho de ser una propuesta docente y de no parecer tan compleja de implementar como otras situaciones que son producto de la investigación. No obstante, hicimos adecuaciones, como incluir la fase de la gráfica, previamente a proponerlas a las docentes. Otras modificaciones se hicieron después, con las maestras. Los propósitos específicos del eje curricular Tratamiento de la información a los que se 
pretendió responder son: generar e interpretar representaciones de sucesos, elaborar una gráfica de barras, y realizar una encuesta.

Metodológicamente, compartimos el doble propósito de las Ingenierías Didácticas de Desarrollo (Perrin-Glorian, 2011), el interés por producir recursos factibles de ser implementados en la enseñanza común, y aportar conocimientos sobre las situaciones mismas. Se planteó la siguiente pregunta de indagación:

¿̇as situaciones elegidas son susceptibles de favorecer, en tanto situaciones adidácticas (categoría de la TSD), el aprendizaje de los aspectos previstos sobre tratamiento de la información, a saber: la representación de información para comunicar un procedimiento, la elaboración e interpretación de gráficas para facilitar una elección y el desarrollo de la encuesta como un medio de obtener información?

Responder esta pregunta, implicó analizar tanto las características a priori de las situaciones -ver de qué manera implican al contenido, qué exigen a los alumnos-, como las respuestas que los alumnos dieron, en aras de identificar indicios de lo que aprendieron. También, nos interesó saber si las situaciones resultaban accesibles y adaptables para las educadoras, y si se lograban conservar el sentido de los conocimientos previstos aun con las transformaciones que cada una imprimiría al implementarlas. Esto último lo analizamos detenidamente en otro texto (Laguna y Block, en prensa).

La implementación de la secuencia se llevó a cabo con dos grupos de tercer grado de preescolar en Michoacán, México. El grupo de la maestra Jimena (grupo 2$)^{6}$ asistía a un Jardín de niños con jornada de tiempo completo ( 8 a.m. a 4 p.m.) ubicado en la periferia de la ciudad de Morelia. El grupo de la maestra Emilia (grupo 1) lo hacía en un Jardín de jornada regular (9 a.m. a 12 p.m.) en el municipio de Copándaro de Galeana, ubicado a unos 25 minutos de la capital del estado. Ambos grupos tenían una asistencia diaria de entre 22 a 26 niños. La secuencia fue desarrollada en el periodo de mayo a junio de 2015, a lo largo de seis clases con una duración de entre 30 a 90 min cada una. Todas las clases fueron videograbadas. En el siguiente apartado se presentará el desarrollo de ambas situaciones en cada uno de los dos grupos.

${ }^{6}$ Los nombres de las docentes han sido cambiados. 


\section{RESULTADOS}

\subsection{HACER UNA RECETA: OCASIÓN PARA REPRESENTAR PROCESOS}

\subsubsection{La situación planeada}

En esta situación, los niños expresarían en papel una receta para hacer gelatina. Para ello, debían organizar información generada de sus conocimientos previos y de los aportes de su maestra sobre cómo hacer gelatina, decidir el orden de los pasos en la receta, decidir cómo disponer espacialmente los datos en la hoja y la manera en que convenía representar el procedimiento. Este trabajo requiere seleccionar y ordenar acciones que conforman la receta (calentar 1lt de agua, agregarle un sobre de grenetina, revolver hasta que el polvo se disuelva, distrubuir en vasitos o molde y refrigerar hasta que cuaje), elaborar notaciones relativas a cantidades (número de gelatinas, de cucharadas, de sobres, etc), y comunicar todo ello con claridad, mediante representación notacional escrita, icónica (dibujos) o ambas.

Con esta consideración, buscamos que la situación fuera de tipo adidáctica (Brousseau, 2007), lo cual, en este caso, implica que el conocimiento (elegir, ordenar, representar información) se desarrolla al resolver la situación misma, al tomar decisiones para llegar a una meta bien comprendida, con la menor dirección posible de las docentes. Para la resolución del problema, se previó que los niños tuvieran oportunidades para desarrollar formas no canónicas de representación, evitando así que éstas se impusieran como objeto de conocimiento arbitrario, pues concebimos las notaciones como un medio de solución para alcanzar un objetivo determinado y no como un fin en sí mismas (Martí y Pozos, 2000).

En la TSD se enfatiza que, en una situación adidáctica, no debe ser el maestro la única o principal fuente de validación. ${ }^{7}$ Un proceso de validación implica la retroacción del medio; se busca que el alumno confronte sus procedimientos con el problema mismo o con los procedimientos de otros alumnos. Aquí previmos tres momentos de este tipo; el primero (durante la primera puesta en común), al verificar si las recetas mostraban claramente los pasos para preparar gelatina. El segundo, al preparar la gelatina, ya que ahí se constataría si el

${ }^{7}$ De acuerdo con Block (1991), Brousseau distingue tres clases de validación; empírica, semántica y pragmática. La empírica describe el proceso de probar algo, mostrando que funciona, sin que sea necesario explicar porqué. 
ordenamiento de los datos en la receta ayudaba o no, en la elaboración. Finalmente, al comer sus gelatinas, pues los alumnos podrían apreciar, a partir del cuajado que obtuvieron, si la cantidad de grenetina era la correcta. Los efectos producidos se comentarían durante las puestas en común donde también se esperaban ciertos aportes de las educadoras dirigidos a institucionalizar saberes; es en este proceso donde el docente clarifica los conocimientos expuestos por los alumnos y ayuda a establecer relaciones entre estos y el saber (explícito) implicado (Brousseau, 1994). Algunos de estos saberes son procedimentales: indicar claramente todos los pasos de una receta, poder representar y organizar la información de diferentes formas, etc., otros son conceptuales, como la doble función de los números - para decir cuánto hay, y para ordenar-.

\subsubsection{El uso de representaciones icónicas como medio eficaz para mostrar procesos consecutivos (grupo 1)}

Comunicación de la consigna. La maestra Emilia (grupo 1) tendía a dar consignas apegadas al diseño, cortas y dichas una sola vez. Así lo hizo en esta ocasión:

Maestra: $\quad$ Van a hacer la receta por parejas. Recuerden, esta receta tiene que ser muy clara porque si yo, se la enseño a alguien, la receta, tiene que tener bien claro cómo se hace una gelatina.

(Fragmento de clase. Sesión 1. Grupo1)

Más adelante, veremos cómo el hecho de establecer que el proceso de elaboración de gelatina debía quedar bien claro funcionó como un criterio que guio el análisis de las representaciones de las recetas. Estas acotaciones, dichas en la consigna, son esenciales para el funcionamiento adidáctico de la situación, ya que dan un marco de acción a los niños.

Representación de secuencias de acciones y procesos a través del dibujo. Al seleccionar imágenes para representar un proceso conocido por ellos y organizarlas, los niños pusieron en juego herramientas de más de un sistema notacional. En el grupo de la maestra Emilia (grupo 1), los niños mostraron los pasos de su receta recurriendo a representaciones variadas: dibujos, escrituras, y combinación de ambas. 
Nos parece que, al resolver el problema, los niños usaron mayormente el dibujo por ser un medio más accesible que el escrito (dado su conocimiento incipiente de la escritura), y porque les facilitaba mostrar los procesos consecutivos que la tarea demandaba. Estos dibujos no eran ornamentales, tenían la función de aportar información. Lo anterior es visible en las coincidencias de los íconos elegidos por distintos equipos para representar los pasos de la receta: calentar el agua (ollas, humo, fuego, estufas), verter la grenetina y disolverla (sobres de gelatina, polvito cayendo, cucharas), y distribuirla en vasitos (jarras, vasos). Un paso complejo de representar, como el de llevar la gelatina al refrigerador para que cuaje, fue resuelto siempre con el dibujo de un refrigerador [figura 1].

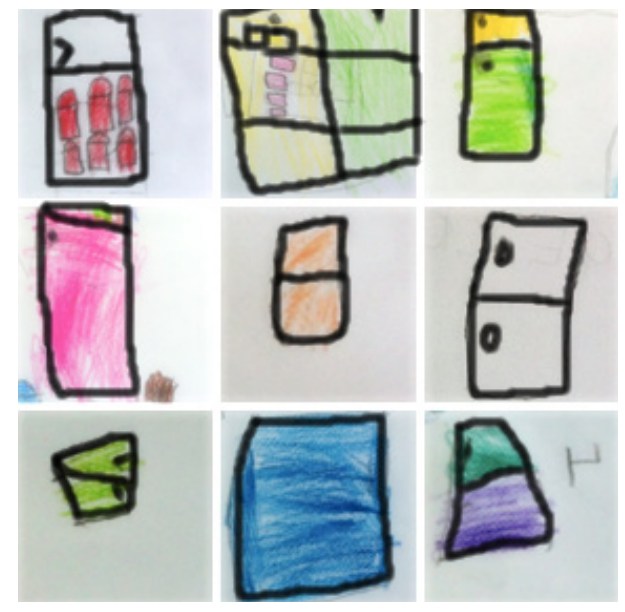

Figura 1. Representación del proceso de cuajado de gelatinas.

Las características de los refrigeradores variaban (cerrados, abiertos, transparentes, sólidos), no obstante, el contexto aportado por la tarea (hacer recetas de gelatina) permitió a los niños interpretar este y otros pasos. Un ejemplo de esta interpretación puede verse en la figura 2. 

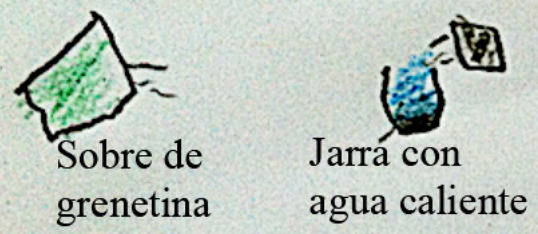
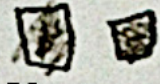
Vasos con gelatina

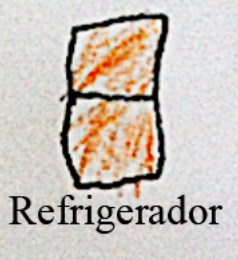

Figura 2. Formato de receta, Alexis.

Aunque los dibujos son simples y, en algunos, no había otros elementos de apoyo como letras o números, los demás niños pudieron interpretar la receta. Dicha comprensión se facilitó por las coincidencias en el tipo de representación, y porque los niños ya asumían que el orden en el papel de izquierda a derecha -que es el de la lectura- expresaba el orden temporal de las acciones. Por otro lado, no todos los niños usaron el dibujo como única forma notacional; destacamos el resultado de Lázaro y Lupita [figura 3] quienes utilizaron escritura y numerales.

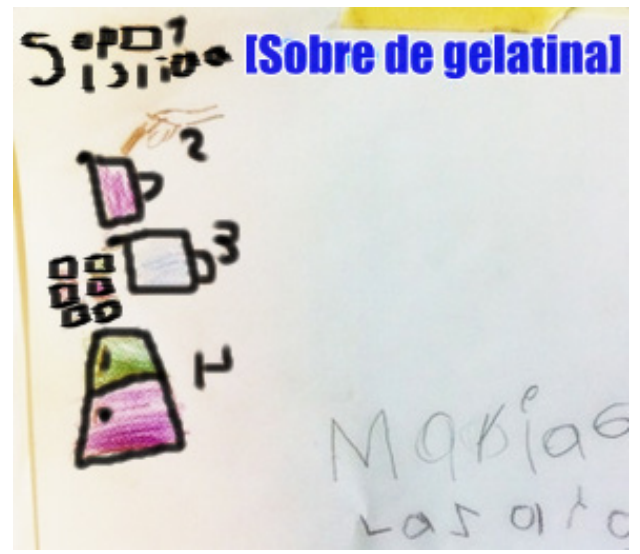

Figura 3. Formato de receta Lupita-Lázaro.

Casi al finalizar su receta, Lupita comentó la falta de ordenamiento numérico: "Ni le ponemos, ni paso uno, ni paso dos, ni paso tres, ni paso cuatro, ni paso cinco" [se está refiriendo a lo que omiten]. Lázaro contestó: "Deja le 
pongo paso y paso" y distribuyó las etiquetas numéricas ordinales de arriba hacia abajo, comenzando desde 1 . Es común que, cuando los niños utilizan notaciones para comunicar - una receta en este caso-, recurran a otro sistema para explicitar mejor el mensaje (Tolchinsky y Karmiloff-Smith, 1993). Además, al leer su receta, los alumnos diferenciaban el uso ordinal de los números y no lo confundían con su función cardinal.

Ubicación de los datos en el micro espacio de la hoja. Durante sus primeros años de escolaridad, uno de los conocimientos y habilidades que los alumnos deben desarrollar es el de la ubicación espacial (Salin, 2004). El micro espacio de la hoja constituye uno de los recursos al alcance de la enseñanza escolar para propiciarlo (el macro espacio, la calle o el campo, son más poderosos pero difíciles de usar en las clases). La situación de la receta dio lugar a un trabajo sobre este aspecto, ya que una decisión importante al organizar información -derivada de la exigencia de claridad- fue el acomodo de los datos en el papel.

En este grupo, casi todos los formatos de receta presentaban una organización de izquierda a derecha (direccionalidad), y de arriba hacia abajo (linealidad), estas características de organización son tomadas del sistema escrito. Los niños se familiarizan con estas convenciones desde temprana edad en prácticas de escritura de su cultura (Brenneman et al., 1996). El ordenamiento dado a los registros permite que, una receta como la de Alexis [figura 2] pueda ser interpretada y saber, a pesar de la ausencia de numerales, cuál es el primer paso, el segundo, y así sucesivamente. En el caso de Lupita y Lázaro [figura 3], este ordenamiento fue, además, apuntalado por el uso de números ordinales. Para otros niños, probablemente la socialización de procedimientos como el de Lupita y Lázaro constituye una ayuda para conocerlos y, eventualmente, usarlos.

Interacciones entre los alumnos. Para decidir qué tipo de notación privilegiar, algunos niños impusieron una forma sobre otra, otros lo platicaron. Lupita y Lázaro por su parte, discutieron sobre el orden de los pasos pero no sobre cómo representar, más bien, fueron tomando decisiones en el proceso. Debido a las decisiones in situ, la escritura de Lupita (sobre de gelatina) quedó como el paso número uno del procedimiento [figura 3], aunque inicialmente pretendía hacer una lista de ingredientes. Este ejemplo deja ver que la organización y distribución del trabajo entre los niños es flexible y que las decisiones que se toman están motivadas por criterios no solamente inherentes al problema que se quiere resolver, sino también por criterios de índole social. En situaciones donde 
prevalece el carácter adidáctico se posibilitan este tipo de interacciones, pues los niños atienden menos, durante un momento, a la aprobación de su maestra. ${ }^{8}$

El papel de las puestas en común. El criterio de claridad en los pasos ayudó para discutir si la información mostrada comunicaba el procedimiento para hacer gelatina. Un ejemplo del uso de este criterio se muestra enseguida:

Maestra: $\quad$ yo les dije: "La receta tiene que ser clara para cualquier persona que la vea", custedes le entienden a todas las recetas que están aquí?

Daniela: $\quad$... no están claras.

Maestra: No están claras... ¿En cuál receta sí nos queda clara la información? Observen las recetas.

Sandra: $\quad$ En la de nosotras... porque pusimos la lista de lo que se ocupa.

(Fragmento de clase. Sesión 1. Grupo1)

A partir de ahí, se observaron las producciones para ver si eran comprensibles para otros. Además de analizar la claridad, en la puesta en común también se compartieron formas notacionales en común y se compararon formas distintas de representar y acomodar [figura 1]. Este momento permitió contrastar dos sistemas notacionales - dibujo y escritura-, hablar de la importancia de la distribución espacial de los datos, y del funcionamiento de numerales en la receta, entre otros. La puesta en común constituyó una primera oportunidad para validar, hacerse de herramientas notacionales e ir creando un sistema compartido que serviría en registros como la encuesta.

La validación de la receta al hacer y al probar la gelatina. La elaboración de gelatina, además de ser la meta para los alumnos, se previó como un momento de validación. Si los niños mostraran dificultades para comprender la receta, se podría acudir nuevamente al criterio de claridad para cuestionarse sobre elementos faltantes o elementos no funcionales en el formato. En el grupo 1, este momento se vio modificado cuando la educadora decidió que todos los niños fueran haciendo juntos la gelatina. ${ }^{9}$ Si bien, esta transformación debilitó la

${ }^{8}$ El estudio de los efectos de la interacción social en el aprendizaje ha sido abordado desde otros enfoques, sobre todo desde aproximaciones socioculturales inspiradas en la obra de Vigotsky (Coll y Onrubia, 1992).

${ }_{9}^{9}$ Más adelante, en entrevista, la educadora comentó que vio la necesidad de guiar la actividad para mantener cierto orden debido al uso de agua caliente. 
validación prevista, los niños sí consultaron con frecuencia la receta para poder decirle a la maestra el paso que seguía.

En otro momento, cuando los alumnos recibieron su gelatina ya hecha, notaron que la única cuajada era la de Lupita. Este suceso los llevó a discutir sobre cómo se comprendía la información de los formatos, específicamente sobre la medida de 1 cucharada de grenetina por vaso. Los argumentos giraron en torno a si una cucharada era suficiente, el tamaño de cuchara que se debería usar, la cantidad de grenetina que se ponía en la cuchara, etc. Así se reavivó la validación que esperábamos, y que es esencial para orientar los esfuerzos de un momento adidáctico: los niños analizaron la forma en que se entiende la información y surgió la necesidad de ser más específicos y claros respecto a la forma de anotar una medida en futuros formatos. Los procesos de validación empírica posibilitan la identificación de errores por los propios alumnos y permiten la evolución de procedimientos. Es decir, tienen la bondad de hacer más independiente el juicio de los alumnos del de su maestro. No obstante, no siempre son factibles de organizar, o el resultado no es siempre el esperado (Block, 1991).

\subsubsection{Uso y funcionalidad del sistema de escritura como herramienta para representar acciones y procesos (grupo 2)}

Comunicación de la consigna. En este grupo, la educadora indicó que, en parejas, harían una receta para hacer una gelatina, y nombró a los integrantes de las binas. Más adelante, mientras los niños trabajaban, la docente fue estableciendo consideraciones que se habían previsto para la consigna: "que se entiendan bien los pasos" y que, "si sus mamás la leen -la receta-, puedan saber cómo hacer la gelatina". Estas últimas precisiones eran necesarias para que los niños tuvieran un eje de referencia al decidir qué poner en su texto y qué no.

Preponderancia del sistema escrito. El hecho de usar la escritura para hacer su receta, llevó al grupo de la maestra Jimena a entrar en un proceso de edición y corrección de textos que generó discusiones sobre el ordenamiento de los datos y la funcionalidad del formato. Las posibles razones de la prevalencia del uso del sistema escrito están relacionadas con la interpretación de la docente de los programas oficiales, y con particularidades de su práctica (abordamos este aspecto en Laguna y Block, en prensa).

Tomaremos el caso de Jenifer y Juan para ilustrar este recorrido. Ambos niños se encontraban en distintos niveles de escritura y se organizaron por 
turnos para escribir los pasos de la receta. Además de la escritura, recurrieron al sistema notacional matemático para dar orden a los pasos. Su primer texto fue el siguiente:

1 i E o E R o i o (primero poner el polvito) [escrito por Juan]

2 Enunaolla [escrito por Jenifer]

3 a E O P E q U a (calentar el agua) [escrito por Juan]

4 EcHarElaGuaenlaolla [escrito por Jenifer]

5 E M E R a E i (meter al refri) [escrito por Juan]

Las oraciones del texto contrastan: Juan, quien se encuentra en un nivel silábico-alfabético (Nemirovsky, 2000), escribe utilizando una grafía por sílaba, alternando mayúsculas y minúsculas. Jenifer en cambio, escribe de manera convencional, sin separar las palabras.

Interacciones y ayudas entre los niños. La tarea encomendada requería de un momento de planificación y discusión. En nuestro ejemplo, Jenifer y Juan antes de poner cada paso, comentaban cuál seguía, y decidían el texto. Jenifer, a diferencia de lo que solían hacer otros niños, no le comentó a su compañero que sus formas de escritura no estuvieran correctas o entendibles. Tampoco se observó a Juan solicitar la ayuda de su compañera o de la maestra para escribir. Otras parejas en cambio, tenían constantes discusiones sobre cuáles letras usar, cómo se escribían ciertas palabras o incluso, algunos niños, con mayor dominio de las características del sistema de escritura, hacían casi todo el texto. En este grupo, se observó que las primeras interacciones de los niños estaban más centradas en las formas de escribir que en el ordenamiento de datos, sin embargo, eso cambió en las siguientes etapas, como veremos.

El papel de las puestas en común en la edición de textos. Al revisar las recetas, el grupo se involucró en un análisis de textos mediante la comparación entre algunas recetas seleccionadas por la maestra. Este momento dio lugar a una primera validación del formato. Como no había variedad en la forma de representar (todos recurrieron al texto), la discusión se centró en el criterio de claridad en la comunicación de los pasos. Derivado del análisis, algunos niños comenzaron a señalar que había recetas con información incompleta o que no se entendía cada paso. En ocasiones, las puestas en común son un espacio para el surgimiento de nuevos problemas (Quaranta y Wolman, 2003). Así, surgió la necesidad de revisar toda la receta, decidir qué modificaciones se 
requerían, y en consecuencia, insertar datos faltantes en una sucesión de pasos ya establecida.

Insertar datos faltantes en el micro espacio de la hoja. Para hacer las modificaciones que querían, los niños tenían que insertar datos en un texto ya organizado según la estructura del sistema escrito: de arriba a abajo y de izquierda a derecha en renglones. En el caso que seguimos, Jenifer y Juan querían agregar el paso de revolver y este tenía que ser colocado dentro de un texto que ya estaba acomodado y numerado. La maestra ubicó a los niños de acuerdo a los cambios que harían para que pudieran apoyarse. Jenifer (Juan no asistío ese día) se sentó con Emiliano quien iba a insertar el mismo paso que ella. La primera dificultad que enfrentaron fue determinar en dónde lo agregarían.

\begin{tabular}{ll}
\multicolumn{1}{c}{ Emiliano y Jenifer, diferentes recetas } \\
Maestra: & (...) ¿dónde vas a ponerle? ... ¿dónde irá revolver entonces? \\
Emiliano: & arriba [Emiliano señala su intención de ubicarlo como primer paso]. \\
Observadora: & ¿dónde vas a poner revolver Jeny? \\
Jenifer: & mmmm aquí [señala el final de la oración número dos que dice En una olla]. \\
Observadora: & ¿después de qué? \\
Jenifer: & de ponerle el puntito. \\
Maestra: & a ver, echar el polvo, echar al refrigerador ¿̇en dónde va mover o revolver? Aquí \\
& dice [lee en la receta de Emiliano]: polvo de sabor, calentar agua (...) ¿̇la metes \\
& al refrigerador y luego le mueves? [Emiliano señala el espacio blanco antes de \\
& la oración cuatro que dice: echar el refri] atrás, ándele, ahí póngalo.
\end{tabular}

(Fragmento de clase. Sesión 3. Grupo2)

Con la ayuda de la maestra, Emiliano ubicó el paso: revolver, antes del paso número cuatro que decía: meter al refri. Entonces, decidió que en lugar de agregar un paso más, tenía que borrar uno ya existente para darle lugar al nuevo. Borró el paso tres: calentar el agua, para sustituirlo por el de: revolver. Por su parte, Jenifer había decidido no borrar nada y agregar el texto como parte del paso número dos. Sin embargo, cuando observó la acción de Emiliano, cambió de parecer y sustituyó el paso tres de su receta por la palabra revolver. Aquí vemos cómo las decisiones de los pares inciden en la manera en que los niños terminan resolviendo. Revolver quedó entonces, antes de poner el agua, lo que llevaría a mover el polvo seco en la olla. Este problema fue retomado por la maestra durante la puesta en común. 
Maestra: (...) algunos niños, como Aitana y Néstor, que agregaron el paso de revolver no borraron otros pasos...pero... Emiliano y Jenny borraron un paso... ¿̇cuál paso borraron para poner el de revolver?

Jenifer: la del agua [paso número tres].

Maestra: $\quad$ el de vaciar el agua cientonces no tienen que vaciar el agua? [Jenifer asiente con la cabeza] ¿entonces, por qué lo borraron? Vamos a ayudarlos entre todos cilistos? [lee en la receta] Dice: primero poner el polvito, en una olla [la maestra reestructura la frase: van a poner el polvito en una olla], revolver, echar el agua en la olla y meter en el refri ¿sí está bien o no?

Iker: $\quad$ no, les faltó calentar el agua (...)

Emilio: $\quad$ iya sé! (...) iRevolver es el de abajo!

(Fragmento de clase. Sesión 3. Grupo 2)

La edición implicó que los niños tuvieran que pensar cómo incorporar información nueva en el micro espacio de la hoja, sin que se perdiera el sentido, la claridad y el acomodo de los pasos ya establecidos. El reto no fue menor, dio pie a que los alumnos se involucraran en el análisis de datos y a que se ayudaran para lograr la tarea con éxito. Este fue un ejemplo, hubo otros, de cómo la educadora organizó una fase -la edición de textos- a partir de sus consideraciones personales, y de cómo esto tuvo un impacto positivo en el desarrollo de la situación y, en consecuencia, en el propósito de enseñanza.

La validación de la receta al hacer y al probar la gelatina. Inicialmente, la maestra Jimena planteó la elaboración de la gelatina conforme a lo previsto en el diseño. Incluso, sugirió a los niños que pusieran la receta sobre la mesa para que pudieran leerla con facilidad. Sin embargo, poco a poco la educadora fue involucrándose en la actividad y terminó ayudando a poner la grenetina y el agua caliente, ${ }^{10}$ en consecuencia, lo que quedó a cargo de los niños fue disolver la mezcla, y así, la posibilidad de validar la receta se vio afectada considerablemente. Lo mismo ocurrió al día siguiente, las gelatinas cuajadas no presentaban diferencias considerables pues al no seguir sus propias recetas algunos posibles errores no surgieron. Como hemos dicho, hay distintos factores que pueden afectar las validaciones empíricas, en este caso, las dos posibilidades de

${ }^{10}$ El hecho de utilizar agua caliente, llevó a ambas educadoras a tomar decisiones que mermaron los procesos de validación previstos. 
validación, la elaboración de gelatinas y la revisión del producto obtenido, se vieron disminuidas por las intervenciones de la docente.

En síntesis, las interacciones y reflexiones derivadas de las recetas dieron pie a que los niños fueran destacando las características que debían tener los formatos para que pudieran cumplir su función, a saber: tener los pasos básicos para que la receta pueda hacerse, respetar cierto orden, y cuidar que ambos aspectos queden claramente expresados. Estos mismos elementos estuvieron presentes en el grupo 1, pero se manifestaron de manera diferente, pues los pasos se representaron mayoritariamente con dibujos. Los resultados -sin dejar de considerar las diferencias en los tipos de representación que generaron en cada grupo, así como las formas en que cada docente compensó las dificultades para llevar a cabo la validación empírica-, son indicadores, nos parece, de las potencialidades de la situación.

\subsection{HACER UNA ENCUESTA: APRENDER QUÉ PREGUNTAR Y CÓMO}

\subsubsection{La situación planeada}

Para la situación de las encuestas, se planteó a los niños la posibilidad de preparar gelatinas para compañeros de otro grupo. Con este fin, equipos de tres o cuatro alumnos debían averiguar la elección de sabor (entre dos opciones) de cada niño de un equipo similar en número del grupo encuestado. La intención fue dar lugar a la realización de una encuesta, desde el diseño del formato hasta el uso de los datos recabados. Para resolver el problema, los niños podrían recurrir a la combinación de sistemas notacionales y usar numerales, marcas de conteo, íconos o escritura. La distribución de los datos -relación de la variable niños con la variable sabor- sería el reto mayor: podría ser a modo de listado o estar por columnas y renglones, acercándose a un formato de tabla.

Una vez hecha la encuesta, los alumnos reportarían a la educadora cuántas gelatinas necesitarían de cada sabor para que se compraran los sobres necesarios. Esto constituiría un primer momento de validación del formato. Con la información ya concentrada, se organizaría una puesta en común (segundo momento de validación) para analizar la funcionalidad de los formatos e identificar los más eficaces. El tercer momento para validar ocurriría al entregar las gelatinas. En esta instancia se podría verificar si se contaba con la información necesaria para asignar a cada niño la gelatina del sabor 
que pidió (relación de variables niños-sabor). La experiencia permitiría la institucionalización de la encuesta como una manera de obtener datos que no se tienen y se destacarían las características que debería reunir un formato para cumplir con su función eficazmente.

Congruentes con el enfoque que nos interesa, se pretendió que las características anteriores permitieran a la situación funcionar como adidáctica, esto es, que favorecieran la participación autonóma de los niños en las tareas, sin que las educadoras propusieran formas específicas de registro. En este apartado, intentaremos demostrar que la encuesta puede llegar a ser una actividad muy fecunda por sí misma, por lo que merece la pena explorarla más ampliamente en estudios didácticos. Como en el apartado anterior, mostraremos lo sucedido en cada uno de los dos grupos.

\subsubsection{Recursos notacionales para representar la relación entre dos variables: nombre del encuestado/sabor de preferencia (grupo 1)}

Presentación del problema. Para introducir la encuesta, se esperaba que las maestras plantearan un problema: hay que saber qué sabor de gelatina -entre dos opciones- escoge cada niño del otro grupo. La maestra Emilia adelantó una solución en la consigna: "Les quiero proponer que vayamos a preguntarle a los niños... cuál sabor prefieren". Por lo tanto, cuando preguntó cómo hacerle, los niños respondieron que yendo a preguntarles. A pesar del tropiezo inicial, la docente rescató el problema cuestionando: "Preguntar qué" y "cómo anotamos lo que digan". Así, los niños lograron proponer formas de preguntar y registrar. Al tener experiencias previas con la receta, las sugerencias de ciertas notaciones salieron de inmediato: anotar con números, escribiéndolo, o con palomitas $[\sqrt{ }]$. Esto se fue especificando al organizarse cada equipo: "Si quiere de limón, ponemos de limón, o le escribimos limón, o asi" -Rey Jesús-. De esa manera, cada equipo fue configurando los registros que veremos enseguida.

Combinación de íconos y escrituras para representar una relación entre variables. Al resolver un problema, los niños tienden a aprovechar aquellas características del medio notacional que mejor satisfacen sus propósitos de comunicación (Tolchinsky y Karmiloff-Smith, 1993). En este caso, el reto de relacionar dos variables (niños y sabores) llevó a los niños del grupo 1 a combinar formas notacionales. Analizaremos, en primera instancia, la manera de representar sabores [figura 4] pues fue una variable presente en todos los formatos. 


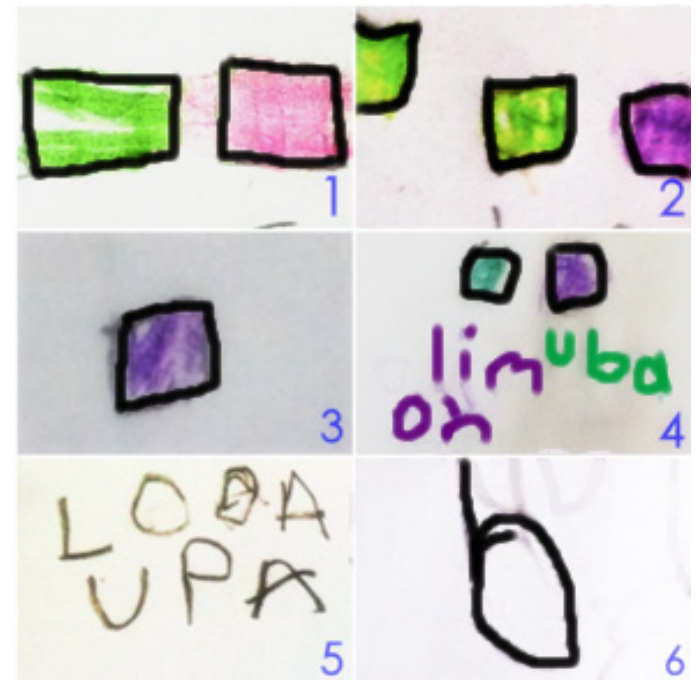

Figura 4. Representación de la variable sabor.

La necesidad de dar cuenta del sabor (dato no tangible, abstracto) llevó a los niños a buscar recursos de representación asequibles. El uso del color fue uno de ellos: verde para limón, y morado o rosa para uva [figura 4]. Los equipos del uno al tres hicieron rectángulos coloreados para representar vasitos con gelatina, mientras que el equipo cuatro puso cuadrados coloreados que representaban directamente el sabor y, al momento de encuestar, escribieron el sabor con colores, aunque no hacían correspondencia con el color del sabor. De acuerdo con Brenneman et al., el uso de colores es más frecuente en la representación icónica porque el color queda fuera de las características del sistema escrito (1996), y también, del sistema numérico. No obstante, en el caso de estas representaciones, el uso del color se integra bien con el uso de escritura.

Por otra parte, no toda representación icónica fue funcional. El equipo seis también representó con dibujos; limón [círculo con línea] y uva [círculo], pero sin referente del color. En este caso, los integrantes del equipo sí distinguían las frutas dibujadas, pero no así los otros niños, ni nosotros.

Para representar a los niños encuestados, todos recurrieron a la escritura del nombre [figura 5]. Sin embargo, hubo variación en las condiciones en que esto se dio. Si bien, algunos niños se decidieron por la escritura de nombres por considerarla mejor opción debido a su conocimiento del sistema escrito (la 
escritura sirve para representar identidad) (Tolchinsky y Karmiloff-Smith, 1993), otros más lo hicieron a partir de la revisión de los formatos en la puesta en común, como veremos más adelante.

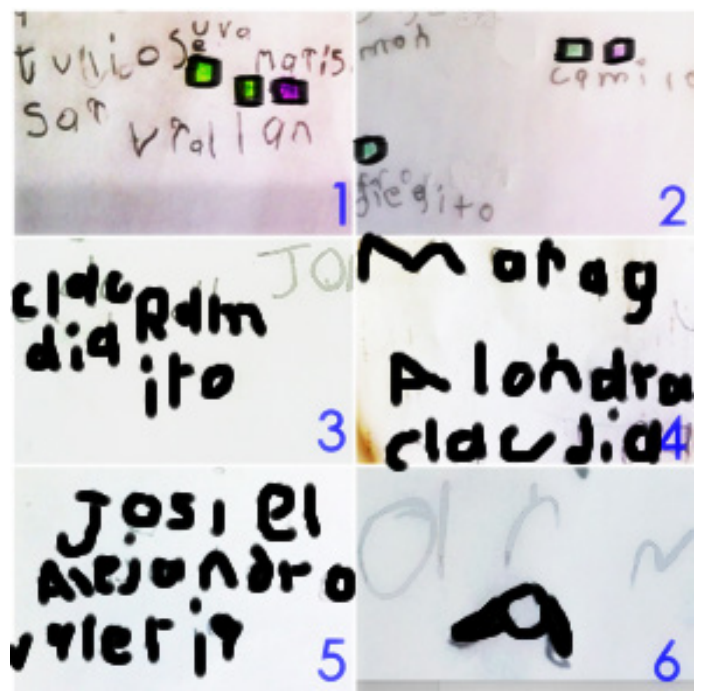

Figura 5. Representación de la variable niños encuestados.

La variedad de formas de hacer la encuesta es consecuencia del funcionamiento adecuado de una fase adidáctica: Ios niños tuvieron espacio para planificar con libertad y autonomía la manera de hacer su formato. En la planificación, algunos equipos lograron ponerse de acuerdo en la pregunta y en quién registraría. El equipo cinco, además, previó la representación de los sabores; limón [LOOA] y uva [UPA] [figura 4]. En general, los niños demostraron una capacidad para establecer un plan de acción de acuerdo a su dominio del conocimiento notacional y al tipo de tarea (Brenneman et al., 1996). En propuestas de tipo adidáctico, estos momentos para pensar y planear son esenciales.

Organización de datos en el micro espacio de la hoja para relacionar variables. Nuevamente, el conocimiento de ciertas pautas de organización convencional en textos (linealidad y direccionalidad), ayudó a que los niños lograran una distribución espacial que les permitiría tener éxito en la relación de variables [figura 6], ya fuera asignando el sabor al nombre del encuestado, o el nombre del encuestado al sabor de gelatina. Aun así, no todos los equipos lograron 
establecer con claridad dicha relación: los equipos dos y cinco [figura 5], no registraron la variable niños encuestados de manera inicial, y el equipo seis [figura 6] distribuyó el listado de nombres de forma independiente al sabor. Estas decisiones mermaron la funcionalidad de los formatos y, en consecuencia, nutrieron las posteriores validaciones.
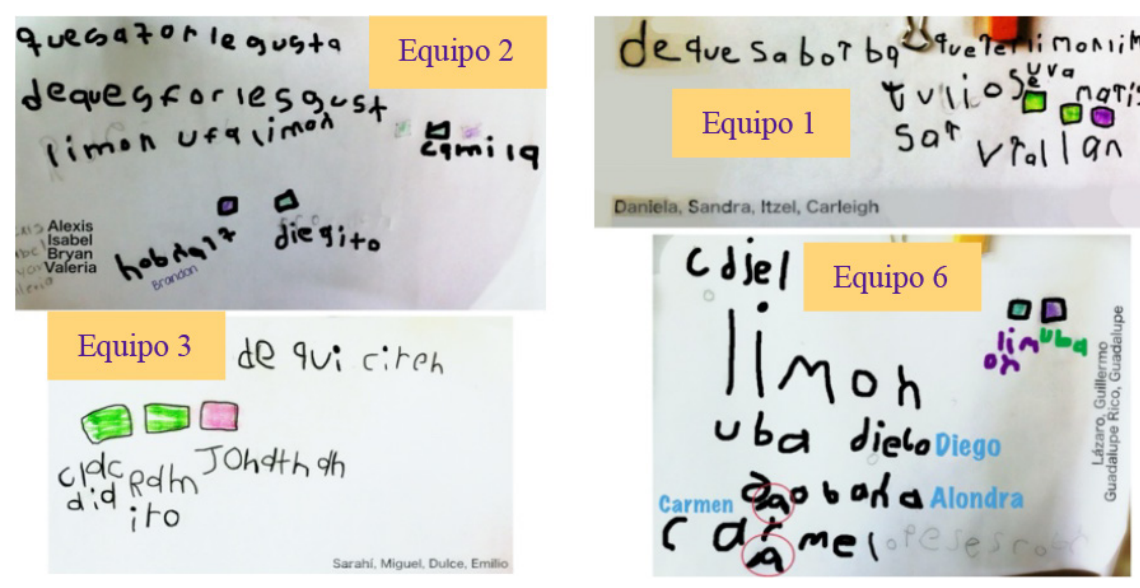

Figura 6. Formatos de encuesta con relación de variables.

En este grupo, la combinación de sistemas notacionales y el acomodo de datos en la hoja resultó efectiva para obtener y relacionar datos. Además, destacamos que cuatro de los seis equipos agregaron la pregunta de la encuesta a su formato, lo cual los acerca a una estructura más convencional y deja ver, a la vez, la influencia de los conocimientos previos de los niños. Se infiere también que lograr mayor economía y claridad no siempre es la única razón por la que los niños deciden incorporar ciertas formas de registro, ${ }^{11}$ sino que la construcción de formatos y el uso de ciertas notaciones están mediados por componentes de interacción social y cultural.

11 Esto mismo lo hemos corroborado en otra situación didáctica, "Los dados de colores", donde señalamos que, en sus registros de puntos ganados, los niños cambiaban sus formas de notación debido a dos tipos de influencia de los compañeros: "las que apuntaban a una cuestión de funcionalidad en el registro, y las que atendían a aspectos de socialización como estatus, liderazgo o rivalidad entre los equipos o secretarios" (Laguna, 2016). 
Comunicación de los datos obtenidos: identificar carencias y negociar soluciones. La maestra solicitó que los equipos dieran la información obtenida en la encuesta mientras ella la iba anotando en el pizarrón (primer momento de validación), y de una vez, aprovechó para ir analizando los formatos de encuesta (segundo momento de validación). Para ello, cada equipo decía cuántas gelatinas requería de cada sabor y explicaban cómo habían hecho su registro, quién había anotado, si hubo organización previa y cómo fue la pregunta. Todos los equipos lograron comunicar la cantidad de gelatinas de cada sabor que les habían pedido, pero no todos lograron explicar a quienes correspondían esas gelatinas.

Las carencias en la representación de la relación que debía establecerse entre encuestados y sabores permitieron discutir sobre cómo establecer o clarificar dicha relación. En algunos casos (equipos dos y cinco) la educadora indujo la escritura de los nombres, a pesar de la resistencia del equipo cinco que no lo veía necesario. El equipo seis [figura 5 y 6], por su parte, tenía el problema de tener el listado de nombres separado de los sabores. Coincidentemente las niñas habían elegido uva y los niños limón, así que, lo resolvieron poniendo un símbolo al nombre de las niñas. Lázaro explicó: le puse carita de niña a las niñas pa' que no me confunda con los nombres. Iniciativas como la de Lázaro dejan ver algunas de las ventajas de que los niños tengan oportunidad de explorar soluciones con sus propias ideas, antes de establecer un recurso convencional.

¿De quién es cada gelatina? Una díficil prueba para los encuestadores. El tercer momento de validación ocurrió en el proceso de la entrega de gelatinas. Inició cuando cada equipo solicitó, registro en mano, la cantidad de gelatinas que necesitaban para hacer la entrega en el otro grupo. Una vez en el aula del grupo encuestado, cada equipo debía entregar las gelatinas conforme a sus datos. En ese momento, los niños tuvieron que recurrir a sus registros para consultar la relación niños encuestados -sabores, la cual cobró así su sentido.

Al volver al aula, se generó un espacio de argumentación sobre la funcionalidad de los registros. El formato fue funcional en la medida en que permitió saber a quién había que entregar cada gelatina, a quién no se le entregó y a quién no se le debió haber entregado. Enseguida mostramos las reflexiones de un equipo que tuvo problemas.

Alina: $\quad$ me confundí porque algunos tenían el mismo nombre o porque no se podía leer bien su nombre.

Ulises: $\quad$ (...) por los que pidieron [niños sin encuestar que solicitaban una gelatina], y unos no vinieron [niños encuestados que no asistieron ese día], por eso se confundieron. 
Rey: maestra, nosotros nos víamos [sic] equivocado, yo traiba [sic] la receta [se refiere a la encuesta]... estaba una niña y le grité a Ulises porque traía una gelatina y me dijo: ia ver!, que si era su nombre porque nos habíamos equivocado [se refiere a la posibilidad de equivocarse al entregar sin ver el registro] y sí era su nombre. Fuimos buscando a otro y a otro, pero sí era.

(Fragmento de clase. Sesión 6. Grupo 1)

Alina y Ulises comentaron dificultades que tuvieron para hacer las entregas: niños que pedían gelatina y no estaban en su lista o niños que coincidían en el nombre. Rey, del mismo equipo, relató cómo lo solucionaron: ubicó visualmente a una niña encuestada por su equipo, le pidió una gelatina a Ulises para ella; él le dijo que revisarían si su nombre aparecía en el registro y así constataron que la información era correcta. El éxito de esa entrega los motivó a repetir esta acción con cada gelatina.

Este es un buen ejemplo de los beneficios de la validación empírica y de la puesta en común; el registro fue el apoyo para verificar las entregas, entonces, su funcionalidad fue validada por el propio equipo: al compartirlo en el aula, el equipo pudo volver sobre sus pasos para describirlos y/o defenderlos, mientras los otros niños, pudieron comprender los procesos del equipo y, posiblemente, apropiarse de algunos procedimientos (Saiz, 1995).

\subsubsection{Construcción grupal de un formato estandarizado para hacer la encuesta (grupo 2)}

Presentación del problema y diseño de un formato en colaboración. La maestra Jimena introdujo el problema de la encuesta de manera contextualizada:

Maestra: me quedaron [gelatinas] cuando fui a comprarlas [muestra dos paquetes de uva y dos de fresa] (...) ¿les gustaría que les hiciéramos una gelatina a los niños del grupo mixto? [dicen que sí] Oigan, pero ¿cómo vamos a saber si los niños, si un niño quiere de uva o quiere de fresa?

(Fragmento de clase. Sesión 2. Grupo 2)

A partir del planteamiento, surgió una discusión sobre cómo obtener el sabor que prefieren los niños del otro grupo. Mientras los niños iban haciendo propuestas, la docente devolvía la información al grupo para analizar su viabilidad. 
Por ejemplo, cuando Iker sugirió realizar la encuesta en equipos, la maestra cuestionó: ¿En equipos de cuántos?, ¿quiénes van?, ¿quién los va a acompañar? Derivado de este momento, se tomaron decisiones colectivas; cada niño encuestaría a otro, anotarían la información en un papel, entre todos decidirían qué diría ese papel, etc.

Esta forma de hacerlo generó que, a diferencia de lo planeado, los alumnos no interactuaran en el nivel de los equipos, sin intervención de la educadora. Aun así, por la forma de la docente de devolver al grupo las propuestas, la mayoría de los niños pudo participar en la construcción del formato que necesitaban. Coincidimos con Sadovsky en que "cuando los alumnos colaboran entre sí para resolver un problema como cuando comparten estrategias de los problemas ya resueltos, los modos de abordar de unos pueden modificar el sistema de decisiones de otros" (2005). De esa forma, los niños quedaron conformes con un formato de encuesta que iba a ser utilizado por todos.

Uso individual del formato grupal, el impacto de las decisiones personales. Aunque la creación y uso del formato se acordó grupalmente, al usarlo cada quien le imprimió su sello personal [figura 7]. En esto puede apreciarse la preservación de la autonomía de los alumnos al tomar decisiones propias sobre lo que consideraban esencial, o no, para el registro, pese a declinar los acuerdos grupales.

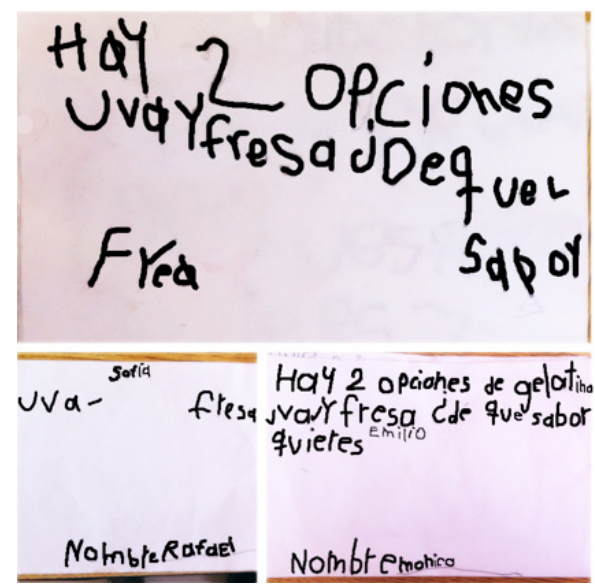

Figura 7. Encuestas individuales Ilenadas de forma distinta. 
En el formato que acordaron utilizar, el nombre del encuestador debía ir en la parte superior con la pregunta: "Hay dos opciones de gelatina, fresa y uva ¿de qué sabor quieres?" La respuesta se debía marcar con una palomita [ $\sqrt{ }$ ] encima de la palabra escrita. El nombre del encuestado iría en la parte inferior. El formato de Emilio (parte inferior derecha) es un ejemplo de cómo se debía hacer la encuesta. En contraste, en el formato superior observamos que el encuestador omitió los nombres, y en lugar de señalar el sabor, lo escribió [frea]. Finalmente, en el formato de Sofía se omite la pregunta, cuando la maestra le cuestionó por qué no la tenía, argumentó que no necesitaba ponerla porque se la sabía bien. Cabe señalar que Sofía fue una participante activa en proponer cómo hacer y usar el formato de encuesta. Estas diferencias dejan ver que, aunque los niños utilicen un formato estándar, se decidirán por los recursos más accesibles para ellos al momento de recabar los datos. Dicha autonomía en las producciones durante las fases adidácticas constituye una condición del sentido de los conocimientos que los alumnos construyen (Panizza, 2003).

Construcción grupal de un formato para vaciar los datos de la encuesta y revisión crítica del uso del formato. Durante el primer momento de validación, la educadora no hizo la tabla para concentrar los datos, en su lugar, cuestionó al grupo sobre cómo podrían saber cuántas gelatinas hacer de cada sabor. Prontamente, Sofía propuso un registro por conjuntos de: “¿Por qué no pones de un lado fresa y del otro lado uva? y le pones con unas flechitas cuántos y cuántos". Iker completó la idea solicitando que la maestra pusiera una rayita en medio, es decir, una línea vertical para separar los conjuntos. También, Sofía sugirió que la educadora fuera preguntando a cada niño de qué sabor le habían pedido y le pusiera un puntito [marca de conteo] según el sabor mencionado. La educadora registró la información conforme a la propuesta de los niños.

De inicio no hubo dificultades pues cada niño leía su propio registro, sin embargo, también tuvieron que leer las encuestas de compañeros que no asistieron ese día, y ahí se evidenció que no todos siguieron el formato al pie de la letra [figura 7]. A partir de ahí, comenzó el segundo momento de validación: Ios niños pudieron discutir sobre los pros y contras de las diferentes formas de llenado, por ejemplo, sobre la dificultad para entender aquellos formatos donde el sabor había sido escrito después de la pregunta, en lugar de haberlo marcado con una palomita $[\sqrt{ }$ ]. El análisis se centró en las dificultades que hubo para usar el formato estandarizado: la falta o ilegibilidad de nombres, o la claridad en el marcado o escritura del sabor. Si bien hubo reflexión sobre las características que debe tener un formato de encuesta, no hubo profundización en 
cuanto a las formas más eficaces de anotar datos, pues esto se había discutido al crear el formato mismo. Aquí, volvemos a destacar la flexibilidad de la secuencia como un atributo que permite modificaciones en el orden de algunos procesos sin hacer que se pierda el sentido de los mismos.

¿Tenemos gelatina para todos? Para el tercer momento de validación, la docente preguntó cómo le iban a hacer para saber a quién entregar la gelatina, a lo que Sofía respondió "Fijándonos... en los papeles". En este grupo, los formatos ya llenos también fueron un referente para los niños en la entrega. A diferencia de lo que pasó en el grupo 1, en este no hubo problemas para saber cuál sabor les habían solicitado y a quién correspondía debido a que los pedidos se hicieron uno a uno. A pesar de que esta organización mermó la función de validación, sí hubo un caso [figura 7] en el que faltó el nombre del encuestado. Se destacó que: 1) había mayor asistencia que el día que se encuestó, 2) en consecuencia, no tenían gelatinas suficientes para todos y, 3) la gelatina que quedaba no se sabía de quién era por la deficiencia del llenado del formato.

Los resultados anteriores permiten afirmar que la situación de la encuesta funcionó en ambos grupos, si bien con modalidades específicas en cada caso. Los cambios a la situación en el grupo 2, en particular que cada quien encuestara a un niño y que todos usaran un mismo formato, debilitaron algunas de sus propiedades, aunque sin perder el sentido de la misma. En el grupo 1 en cambio, se pudieron identificar formas distintas de anotar los datos para jerarquizar cuáles eran mejores por su eficacia; también la cantidad de niños encuestados fue una condición favorable, al hacer díficil recordar el sabor de memoria, con lo que el registro se hizo necesario y útil en la entrega.

\section{CONCLUSIONES}

A partir de los resultados obtenidos, es posible afirmar que, en términos generales, las situaciones de creación y uso de registros que aquí expusimos, dieron lugar a un trabajo fecundo por parte de los alumnos en varios temas del eje conceptual de Tratamiento de la información: representación notacional de procesos, realización de encuestas, tratamiento de la información recabada y toma de decisiones a partir de la misma.

Considerando que fomentar los espacios de invención semiótica donde los niños, sobre todo en edades tempranas, tengan oportunidad de crear nuevos signos y darles significados, debe considerarse como objetivo de enseñanza 
(Goldin, 1998), confirmamos la pertinencia de las situaciones aquí analizadas: los niños exploraron problemas complejos con datos disponibles (receta) y no disponibles (encuesta) que favorecen poner en juego una amplia diversidad de recursos notacionales. Las notaciones creadas por los alumnos se pusieron a prueba, se sometieron a valoración grupal, se vieron sus limitaciones y se jerarquizaron de acuerdo con su eficacia. Es probable que estos aprendizajes permitirán a los niños tener una mayor cantidad de recursos notacionales y organizativos para futuras actividades tanto de tratamiento de la información como de otros aspectos de la matemática escolar.

Como se esperaba ambas situaciones funcionaron, en tanto adidácticas, los alumnos desarrollaron, con relativa autonomía, formas personales de representación y combinaron sistemas notacionales: icónico, numérico y escrito de acuerdo al contexto generado por las demandas de la tarea. Es importante señalar, por otra parte, que las características de la adidacticidad no funcionaron en su totalidad, todo el tiempo, de acuerdo al presupuesto teórico. En principio, se supone que el medio ofrece una retroalimentación que hace evidentes los errores a los ojos de los alumnos. Sin embargo, hubo episodios en los que se pudo observar que una de las docentes, o las dos, no mantuvieron la reticencia (Sensevy, 2011) esperada y, en consecuencia, no dejaron que el medio ejerciera la función de validar o invalidar las resoluciones. Este hecho nos lleva a destacar que, en ciertos momentos, dos características interrelacionadas entre sí -la validación y la reticencia- funcionaron débilmente, o no funcionaron. Un ejemplo de lo anterior ocurrió en el grupo 2, cuando la docente no permitió que las gelatinas elaboradas fueran la prueba contundente del éxito o fracaso del formato de receta. No obstante, la educadora encontró otras formas de dar lugar a cierta validación mediante cuestionamientos bien dirigidos de ella hacia los niños. En el grupo 1 en cambio, la producción de las gelatinas sí permitió detectar un error en la forma de aplicar la receta, faltó grenetina, lo cual puso en evidencia la existencia de un margen en la manera de interpretarla (la unidad "cucharas") y llevó a hacer precisiones (qué tan llenas, de qué tamaño son, etc.). Por ello, que las situaciones contemplen más de un momento para validar y que la validación se pueda hacer de distintas maneras, constituyen características que contribuyen a la flexibilidad de las secuencias y, en esa medida, las robustecen.

En otros momentos del desarrollo de las situaciones, que no analizamos aquí por falta de espacio, pudimos observar que las docentes, con el conocimiento que tienen de sus alumnos, van mediando el tipo de ayuda que brindan a cada uno, es decir, regulan diferencialmente el grado de reticencia que sostienen. De 
acuerdo con las educadoras, algunas de estas intervenciones están relacionadas con el propósito de sostener la estabilidad emocional de aquellos niños que tienen mayores dificultades, lo cual no es tema menor. Goldin señala que en vista de la fuerte evidencia de que las emociones negativas están muy extendidas en relación con las matemáticas, se debe prestar mucha más atención a la psicología del desarrollo del afecto en los estudiantes (1998). El hecho de que los niños estén motivados y se sientan capaces es tan importante en la clase de matemáticas que, a veces, esta necesidad entra en conflicto con la de sostener la resolución autónoma de un problema o la confrontación con sus propios errores. Probablemente, en ciertos casos, esta última debe ceder a cambio de preservar la confianza en sí mismo y la motivación para aprender.

Es oportuno recordar aquí que la adidacticidad constituye una categoría teórica (Sadovsky, 2005; Laguna, 2016), y en ese sentido, describe un ideal didáctico. En la práctica, un medio adidáctico presenta matices. A final de cuentas, se trata de buscar un equilibrio entre situaciones didácticas y adidácticas, de tal manera que los alumnos tengan experiencias de resolución autónoma, y que, al mismo tiempo, las situaciones sigan siendo factibles, manejables por los docentes. Desde ese punto de vista, pensamos que la implementación de la receta y la encuesta constituyen un buen ejemplo: la gestión de las educadoras fue fundamental para permitir que los niños participaran como lo hicieron, desarrollando propuestas, proponiendo soluciones, probándolas, y no ciñéndose a seguir instrucciones.

Para que la resolución autónoma fuera viable, fue importante el tipo de problema en el que se enmarcó la construcción y uso de los registros: hacer gelatinas reales para un determinado grupo de niños. El problema implicó averiguar información en dos sentidos: sobre los niños, quiénes y cuántos eran y qué sabor prefieren; y sobre las gelatinas, cómo se preparan, cuántos sobres comprar de cada sabor, cuántos vasos se requieren, cómo distribuir las gelatinas a sus destinatarios, etc. Dichas condiciones eran posibles porque el proceso mismo genera que ciertas contingencias -como variaciones en la asistencia de los niños encuestados y del propio grupo encuestador- puedan ocurrir y sean parte del problema mismo. Las contingencias, si son aprovechadas para nutrir el problema, permiten ampliar el marco de discusión sobre el formato, validar su uso y pertinencia, así como determinar las características que debe cumplir el formato para que sea efectivo.

Es notorio que las situaciones se reconstruyeron de maneras diferentes en las clases de cada docente, lo que deja ver la huella personal del trabajo de cada una. Este es un resultado en el que profundizamos en otro texto (Laguna y Block, en prensa), destacaremos aquí solamente que las situaciones pudieron adaptarse a 
las circunstancias de cada educadora. La flexibilidad de las situaciones permite que las transformaciones que les imprimieron y las las contingencias generadas por el tipo de problema, sean manejables por las docentes y, de esta forma, aumenta su potencialidad para ser modificadas sin perder su sentido. Por lo anterior, las consideramos candidatas para funcionar en las aulas de enseñanza regular, bajo la conducción de docentes que tengan afinidad con el enfoque didáctico.

\section{REFERENCIAS}

Artigue, M. (1995). Ingeniería Didáctica. En P. Moreno (Ed.), Ingeniería Didáctica en Educación Matemática. Un esquema para la investigación y la innovación en la enseñanza y el aprendizaje de las matemáticas (1ra ed.) 33-60. Una empresa docente. Grupo editorial Iberoamérica S. A. de C. V.

Artigue, M. (2011). L'ingénierie didactique: un essai de synthèse. Amont et en aval des ingénieries didactiques: 15e École d'Été de didactique des mathématiques, 23(1), 225-237.

Block, D. (1991). Validación empírica del conocimiento en la clase de matemáticas, en la primaria. Cero en Conducta, 25(6), 4-9.

Block, D., Moscoso, A., Ramírez, M., y Solares, D. (2007). La apropiación de innovaciones para la enseñanza de las matemáticas por maestros de educación primaria. Revista Mexicana de Investigación Educativa, 12(33), 263-294.

Brenneman, K., Massey, C., Machado, S. F., y Gelman, R. (1996). Young children's plans differ for writing and drawing. Cognitive Development, 11(3), 397-419. https://doi. org/10.1016/s0885-2014(96)90011-8

Brousseau, G. (1986). Fondements et méthodes de la didactique des mathématiques. Recherches En Didactique Des Mathématiques, 7(2), 33-115. https://revue-rdm. com/1986/fondements-et-methodes-de-la/

Brousseau, G. (1994). Los diferentes roles del maestro. En C. Parra and I. Saiz (Eds.), Didáctica de Matemáticas. Aportes y Reflexiones (1ra ed.) 65-94. Paidós.

Brousseau, G. (2007). Iniciación al estudio de la teoría de las situaciones didácticas (1ra ed.). Libros del Zorzal.

Chevallard, Y. (1991). La transposición didáctica. Del saber sabio al saber enseñado (1ra ed.). AIQUE.

Coll, C. y Onrubia, J. (1992). El análisis del discurso y la construcción de significados compartidos en el aula. Revista Latina de Pensamiento y Lenguaje, 241-259. 
Fuenlabrada, I. (2010). Investigación evaluativa de la implementación del PEP04. Campo de Pensamiento Matemático. Informe final de investigación. 1-69. Secretaría de Educación Pública.

Goldin, G. (1998). Representational systems, learning, and problem solving in mathematics. Journal of Mathematical Behavior, 17(2), 137-165.

Laguna y Block, (en prensa). Reconstrucción de situaciones didácticas de matemáticas en el aula. Un estudio en preescolar. Revista Latinoamericana de Investigación Educativa.

Laguna, (2016). La enseñanza del tratamiento de la información en preescolar. Un estudio sobre procesos de interpretación y reconstrucción de situaciones didácticas. Tesis de maestría, DIE-CINVESTAV-IPN. https://bit.ly/3flbPNn

Martí, E. y Pozos, J. (2000). Más allá de las representaciones mentales: la adquisición de los sistemas externos de representación. Infancia y aprendizaje, 23(90), 11-30. https:// doi.org/10.1174/021037000760087946

Nemirovsky, M. (2000). Antes de empezar: ¿Qué hipótesis tienen los niños sobre el sistema de escritura? En M. Nemirovsky, Sobre la enseñanza del lenguaje escrito... y temas aledaños, 15-25. Paidós.

Panizza, M. (2003). Conceptos básicos de la teoría de situaciones didácticas. En Panizza (Comp.) Enseñar matemática en el Nivel Inicial y primer ciclo de EGB: Análisis y propuestas. Paidós.

Perrin-Glorian, M-J. (2011). L'ingénierie didactique à l'interface de la recherche avec l'enseignement. Développement des ressources et formation des enseignants. En amont et en aval des ingénieries didactiques: 15e École d'Été de didactique des mathématiques, Clermont-Ferrand (Puy-de-Dôme), 23(1), 57-78.

Perrin-Glorian, M.J., Deblois, L., y Robert, A. (2008). Individual practising mathematics teachers. Studies on Their Professional Growth. Mathematics Teacher Education, 35-59.

Quaranta, M. y Wolman, S. (2003). Discusiones en las clases de matemáticas. Qué, para qué y cómo se discute. En Panizza, M. (Comp.) Enseñar Matemática en el Nivel Inicial y primer ciclo de EGB: Análisis y propuestas. Paidós.

Robert, A. (2003) De l'idéal didactiqueaux déroulements réels en classe de mathématiques : le didactiquement correct, un enjeu de la formation des (futurs) enseignants (en collègeet lycée). Didaskalia, 22, 99-116.

Sadovsky, P. (2005). La teoría de situaciones didácticas: un marco para pensar y actuar la enseñanza de la matemática. En Alagia, H., Bressan, A. y Sadovsky, P. Reflexiones teóricas para la Educación Matemática. Libros del Zorzal.

Saiz, I. (1995). ¿Confrontación o corrección?, trabajo elaborado para el curso sobre "La resolución de problemas en la escuela primaria", dictado en la Escuela Normal de San Luis del Palmar, Corrientes, mimeo. 
Salin, M. (2004) La enseñanza del espacio y la geometría en la enseñanza elemental. En Números, formas y volúmenes en el entorno del niño, Universidad Internacional Menéndez y Pelayo de Santander, 37-80.

Sensevy, G. (2011). Le sens du savoir. Éléments pour une théorie de l'action conjointe en didactique. De Boeck.

SEP. (1972). Matemáticas. Primer grado. Auxiliar didáctico. Comisión Nacional de libros de Texto Gratuito.

SEP. (2004). Programa de Educación Preescolar 2004. Secretaría de Educación Pública.

SEP. (2010). El placer de aprender, la alegría de enseñar. Secretaría de Educación Pública.

SEP. (2018). Aprendizajes clave para la educación integral. Educación preescolar: Plan y programas de estudio, orientaciones didácticas y sugerencias de evaluación. Secretaría de Educación Pública.

Tolchinsky, L. y Karmiloff-Smith, A. (1993). Las restricciones del conocimiento notacional. Infancia y Aprendizaje, 62(63), 19-51. https://doi.org/10.1080/02103702.1993.108

MARÍA LAGUNA

Dirección: Departamento de Investigaciones Educativas del Centro de Investigación y Estudios Avanzados (DIE-CINVESTAV-IPN), México, CDMX, columbus_mgrl@hotmail.com

Teléfono: (52) (55) 54832800 ext 1081 